\title{
Population-based patterns and cost of management of metastatic non-small cell lung cancer after completion of chemotherapy until death
}

\author{
Sri Navaratnam ${ }^{\mathrm{a}, *}$, Erich V. Kliewer ${ }^{\mathrm{b}, \mathrm{c}}$, Jim Butler ${ }^{\mathrm{d}}$, Alain A. Demers ${ }^{\mathrm{b}, \mathrm{c}}$, Grace Musto $^{\mathrm{b}}$, Ketan Badiani ${ }^{\mathrm{e}}$ \\ a Department of Internal Medicine, University of Manitoba, Faculty of Medicine, 675 McDermot Avenue, Winnipeg, R3E 0V9 Canada \\ ${ }^{\mathrm{b}}$ Epidemiology and Cancer Registry, CancerCare Manitoba, Winnipeg, Canada \\ ${ }^{c}$ Department of Community Health Sciences, University of Manitoba, Winnipeg, Canada \\ ${ }^{\mathrm{d}}$ Australian Centre for Economic Research on Health, College of Medicine and Health Sciences, Australian National University, Canberra, Australia \\ e Manitoba Institute of Cell Biology, CancerCare Manitoba, Winnipeg, Canada
}

\section{A R T I C L E I N F O}

\section{Article history:}

Received 5 October 2009

Received in revised form

11 December 2009

Accepted 17 January 2010

\section{Keywords}

Lung cancer

NSCLC

Costs

Economic

\begin{abstract}
A B S T R A C T
Background: The aim of this study was to examine the patterns and costs of management of non-small cell lung cancer (NSCLC) after completion of chemotherapy until death in a population of patients in Manitoba, Canada.

Patients and methods: Stage IIIB and IV NSCLC patients diagnosed between January 1997 and June 2000 who received chemotherapy as the primary treatment, completed their chemotherapy and survived for at least 28 days since their last treatment, and were on best supportive care (BSC) were selected. Treatment, services received, costs, and survival were determined by chart review and examining various databases including the Manitoba Cancer Registry, medical claims, hospitalizations, and prescription drugs. Costs of treatment, average cost per patient, and lifetime treatment costs were calculated.

Results: Of the 2463 patients diagnosed with NSCLC over the study period, 150 patients matched our study criteria. From the beginning of the first chemotherapy treatment, the median survival time was 31.8 weeks, while from the date of BSC the median survival time was 13.8 weeks. The average cost per case was $\$ 10,805$ from last date of chemotherapy and $\$ 8654$ during the BSC period. The average cost per patient-month ranged from $\$ 1645$ to $\$ 1792$ in current prices. Lifetime treatment costs ranged from $\$ 8702$ to $\$ 11,057$. Hospitalizations accounted for $80 \%$ of the total treatment costs.

Conclusion: The largest overall component of cost after the end of chemotherapy was hospitalizations. Effective new therapies that reduce the episodes of hospitalizations would have a significant impact on decreasing aggregate costs.
\end{abstract}

Crown Copyright $\odot 2010$ Published by Elsevier Ireland Ltd. All rights reserved.

\section{Background}

Worldwide, lung cancer is the most common cause of cancer death ( $17.8 \%$ of all deaths) [1] and the most common cancer in terms of incidence ( $12.3 \%$ of all cases). Its incidence rate has increased by $20 \%$ since 1990 [2]. This disease can generally be classified into two types based on histology: non-small cell lung cancer (NSCLC) and small cell lung cancer (SCLC). The majority (85\%) of new cases are NSCLC $[2,3]$. NSCLC can be further subdivided into adenocarcinoma, squamous cell carcinoma, large cell carcinoma, and other subtypes [3]. There are well-established guidelines that indicate appropriate therapeutic options for NSCLC patients. Nevertheless, the survival of NSCLC patients is poor, as most individuals have regional or advanced disease at diagnosis; approximately 50-65\% are graded as Stage IIIB or Stage IV [4]. Recently published meta-

\footnotetext{
* Corresponding author.

E-mail address: sri.navaratnam@cancercare.mb.ca (S. Navaratnam).
}

analyses and large randomized trials have clearly indicated that a combination of chemotherapy improves overall survival and quality of life in advanced NSCLC $[5,6]$. Studies that examined the use of agents such as docetaxel and pemetrexed for second line therapy have a survival advantage $[7,8]$. New targeted therapies, namely epidermal growth factor receptor (EGFR)-tyrosine kinase inhibitors such as erlotinib or getfitinib are also utilized for those patients who have failed first or second line chemotherapy [9]. These agents have minimal side effects with modest improvement in survival.

Currently, patients with advanced NSCLC have more options for chemotherapy than ever before. However, more treatment options come at a cost of increasing the burden on the healthcare system. It is consequently important to understand the patterns of treatment of advanced NSCLC, outcomes of treatment, and its cost in a nonclinical trial setting in order to successfully select patients for newer therapies and to effectively introduce these newer options in the treatment plan.

Most cost analysis studies on NSCLC are based on randomized clinical trials of various chemotherapeutic drugs and therefore 
may not accurately reflect the costs associated with a similar chemotherapy treatment in a non-clinical trial setting [10]. Additional costs associated with chemotherapy administration such as those associated with infrastructure, nursing, radiation treatment, hospital admissions, as well as non-contingent costs such as those associated with emergency visits and other health care services are also very important to be included in the analysis. Previously published studies neither included this level of information nor investigated the costs for the period following completion of chemotherapy until death.

This historical cohort study examines the services received by patients with advanced NSCLC from the time they completed chemotherapy until death and provides population-based estimates of costs related to these services. This information is beneficial for improving patient management and appropriately allocating resources.

\section{Patients and methods}

\subsection{Patient selection}

Information on residents of the Province of Manitoba diagnosed with trachea, bronchus, and lung cancer (ICD-9 162) between January 1997 and June 2000 were abstracted from the Manitoba Cancer Registry. NSCLC cases diagnosed with Stage IIIB (with pleural effusions or supraclavicular involvement) or Stage IV tumors were selected for the study. Additional, selection criteria included treatment with chemotherapy and having survived at least 28 days after the end of their chemotherapy. These patients were considered to be on best supportive care (BSC) period.

\subsection{Patient outcome analysis}

Survival was calculated from diagnosis to either death, emigration (derived from the Manitoba Health Population Registry) or end of the study (March 31, 2002). Survival was calculated using the Kaplan-Meier method. Data management and analyses were done using SAS v8.2.

\subsection{Determination of costs}

Direct cost information and service utilization information were extracted from the Manitoba Cancer Registry, OpTx (electronic) chart review, Manitoba Health Population Registry, the Physician Claims database, the Hospital Discharge database, and the Drug Program Information Network (DPIN) database. For the Hospital Discharge database, costs were estimated by multiplying the resource utilization rates by their corresponding unit costs. Direct costs were available from the Physician Claims database and the DPIN database.

For radiotherapy, a cost model was developed dividing a course of radiotherapy into the initial visit $(\$ 102.66)$, the simulation (\$123.30), the treatment planning (\$67.50), the treatment visit(s) (initial visit - \$25.31, subsequent visit - \$29.98), and the followup visits (\$33.03). Costs for each patient were calculated with each of these phases of a radiotherapy session. Not all patients incurred each of these costs. These radiotherapy cost estimates exclude costs associated with quality assurance, capital charges, maintenance of buildings and equipment, and costs of management staff. It should be noted that while the period of the study extends from 1997 to 2001, 2002 costs were utilized in this study since there were no other comparable estimates of cost for radiotherapy. The mean radiotherapy treatment cost for all cases was determined to be $\$ 385.80$ (range $\$ 190.98-\$ 823.94$ ).
Table 1

Alternative estimates of treatment costs. ${ }^{\mathrm{a}}$.

\begin{tabular}{lll}
\hline & Low estimate $^{\mathrm{b}}$ & High estimate \\
\hline From date of last chemotherapy & $C_{i j}^{1}$ & $C_{i j}^{3}$ \\
From date of $\mathrm{BSC}^{\mathrm{c}}$ & $C_{i j}^{2}$ & $C_{i j}^{4}$ \\
\hline
\end{tabular}

${ }^{a} C_{i j}$ is the cost of treatment for the $i$ th person in the $j$ th month.

b Low estimate does not include additional costs. High estimate includes additional costs.

c Start date of BSC is 4 weeks from the last date of chemotherapy.

\subsection{Cost analysis}

The cost of services was calculated for each month of survival until death or end of follow-up (complete information for treatment was not available for 4 patients). The cost was calculated from the commencement of BSC period as well as from the last day of chemotherapy. For each of these two time periods two estimates were determined. A low estimate was determined using cost information from various data and the high estimate was obtained by adding the costs of additional treatment gathered through a chart review. Hence, there are four estimates of treatment cost (Table 1 ).

The mean cost of treatment was calculated per month by summing all costs for the $i$ th patient in the $j$ th month and dividing it by the number of patient-months of follow-up observed in the $j$ th month. Lifetime treatment costs were determined by applying monthly survival probabilities from the Kaplan-Meier survival analysis to the mean cost per patient in each month. This lifetime treatment cost (LTC) estimate is in current prices and not adjusted for inflation. A constant price estimate was determined by converting the costs in each month using the Canadian Consumer Price Index (CPI) for 1998: the same year from which the costs for the hospitalizations were derived. A mid-year CPI was employed and items were not seasonally adjusted. A discounted estimate was also calculated using a $5 \%$ per annum discount rate. Altogether, a total of 12 different series of monthly costs were calculated. Costs were evaluated from the viewpoint of Canada's public health system. All monetary values are expressed as Canadian currency.

\section{Results}

There were 3091 new cases of lung cancer in Manitoba over the study period. Of these, $79.7 \%$ were NSCLC, $11.8 \%$ were SCLC, and $8.4 \%$ were non-specified (Table 2 ). Of the total 2463 NSCLC cases ( $58 \%$ were males and $42 \%$ were females) and only $16.8 \%$ of all 2463 cases, which were of all stages, received chemotherapy.

For the period of the study, we identified 150 NSCLC cases (6\% of all patients with NSCLC) that met our inclusion criteria and considered to be on BSC period. Of these 150 patients, $56 \%$ were male and $44 \%$ were female. Twenty-two percent of patients had Stage IIIb NSCLC while the majority (78\%) had Stage IV NSCLC (Table 2). With respect to the age of the study popu-

Table 2

Morphology of lung cancer by sex, Manitoba 1997-June 2000.

\begin{tabular}{|c|c|c|c|c|c|c|}
\hline \multirow[t]{2}{*}{ Type } & \multicolumn{2}{|l|}{ Males } & \multicolumn{2}{|c|}{ Females } & \multicolumn{2}{|c|}{ Total } \\
\hline & $N$ & $\%$ & $N$ & $\%$ & $N$ & $\%$ \\
\hline NSCLC & 1431 & 80.3 & 1032 & 78.8 & 2463 & 79.7 \\
\hline SCLC & 195 & 10.9 & 171 & 13.1 & 366 & 11.8 \\
\hline Other & 155 & 8.7 & 106 & 8.1 & 261 & 8.4 \\
\hline Metastatic NSCLC & 84 & $56.0^{\mathrm{b}}$ & 66 & $44.0^{\mathrm{b}}$ & 150 & 100 \\
\hline Stage IIIb & 17 & 20.2 & 16 & 24.2 & 33 & 22.0 \\
\hline Stage IV & 67 & 79.8 & 50 & 75.8 & 117 & 78.0 \\
\hline
\end{tabular}

a Metastatic NSCLC: These are patients with Stage IIIb or Stage IV NSCLC who had chemotherapy and were in BSC period. These are eligible study patients.

b Percentage of total number $(N=150)$ of eligible study patients. 
Table 3

Survival of NSCLC patients $(N=150)$.

\begin{tabular}{|c|c|c|c|c|}
\hline Time & Median (weeks) & $95 \% \mathrm{Cl}^{\mathrm{a}}$ & Survived 1 year (\%) & Survived 2 years (\%) \\
\hline From date of diagnosis & 40.6 & $35.4-45.7$ & 30.7 & 11.7 \\
\hline From first date of chemotherapy & 31.8 & $28.7-37.7$ & 26.7 & 8.9 \\
\hline From date of BSC & 13.8 & $10.9-16.3$ & 16.8 & 5.8 \\
\hline
\end{tabular}

a $\mathrm{CI}$ : confidence interval.

Table 4

Summary of services provided to BSC NSCLC from last date of chemotherapy.

\begin{tabular}{lrrr}
\hline Service & Number & Number/person & $\begin{array}{l}\text { Number/100 } \\
\text { person weeks }\end{array}$ \\
\hline Medical claims & 4353 & 29.8 & 112.3 \\
Prescription drug use & 3050 & 20.9 & 78.7 \\
Tests & 729 & 5.0 & 18.8 \\
Hospitalizations & 271 & 1.9 & 7.0 \\
Drugs & 259 & 1.8 & 6.7 \\
Procedures & 144 & 1.0 & 3.7 \\
Physician visits & 135 & 0.9 & 3.5 \\
Radiotherapy & 113 & 0.8 & 2.9 \\
Emergency visits & 65 & 0.5 & 1.7 \\
Outpatients & 35 & 0.3 & 0.9 \\
Total & 9154 & 62.7 & 236.2 \\
\hline
\end{tabular}

a These are drugs for which prescriptions were not filled but were provided as in-patients.

lation, $47.6 \%$ of males and $34.9 \%$ females were over 65 years of age.

All the cases selected for the study had first line chemotherapy and $16(10.7 \%)$ also received second line treatment. One hundred and forty cases died before the end of follow-up and two emigrated out of the province. The median survival time was 40.6 weeks from the date of diagnosis (Table 3 ). The proportion of patients who survived one and two years were $31 \%$ and $12 \%$, respectively. From BSC date, the median survival time was 13.8 weeks.

A summary of services utilized by NSCLC patients from the date of last chemotherapy is listed in Table 4. Claims for fees for services provided by physicians and nurse practitioners (medical claims) ranked as the highest service utilized, which was followed by prescription drug use. On average 29.8 medical claims were made per case (29.4 claims for males 30.3 claims for females). Among males, those aged 65 years or older had the most claims (34.0 claims), while among females, the most claims were observed in the age group between 55 and 64 years (36.0 claims). Most of these claims
(64.7\%) were related to people's cancers and 6.7\% were for illdefined causes. Each person on average had 29.8 medical claims or 1.12 medical claims per person week or 112.3 medical claims per 100 person weeks (Table 4). Expressing the next three highest services utilized in a similar manner (Table 4), there were 0.787 prescriptions per person week, 0.18 tests per person week, and 0.07 hospitalizations per person week. The frequency of the utilization of these medical services is perhaps better represented as 1 medical claim per 0.89 person weeks, 1 prescription per 1.3 person weeks, 1 test per 5.5 person weeks, and 1 hospitalization per 14.3 person weeks of follow-up.

The NSCLC cases bought a total of 3050 prescriptions for an average of 20.9 per patients (Table 4). The average number of filled prescriptions was similar for males and females. The majority $(41.7 \%)$ were filled for central nervous system drugs, most frequently for morphine and codeine. This was followed by prescriptions for hormones and substitutes $(11.7 \%)$ and cardiovascular drugs (11.5\%). The third most frequently prescribed drug was dexamethasone (4.6\%).

The patient cohort had a total of 271 hospitalizations, 1.9 hospitalizations per person (Table 4). There were 21.2 hospital-days/male and 25.2 hospital-days/female (total of 23.0 hospital-days/person). These hospitalizations were more frequently related to the cancer $(55.4 \%)$ or convalescence and palliative care (10.7\%).

Forty-six percent of the 150 patients had at least one procedure performed. Transfusion of packed cells was performed on $10.3 \%$ of these patients. The most common test performed was a CAT scan of the head in $11.5 \%$ of patients. This information was captured through chart review.

Hospitalization episodes accounted for approximately $80 \%$ of the total costs related to the study cases (Table 5). They were followed by the costs for physician visits. The total cost from the last date of chemotherapy was $\$ 1,577,520$ while the total cost during the BSC period was $\$ 1,263,493$. The difference between these

Table 5

Costs used in analysis $(N=146)$.

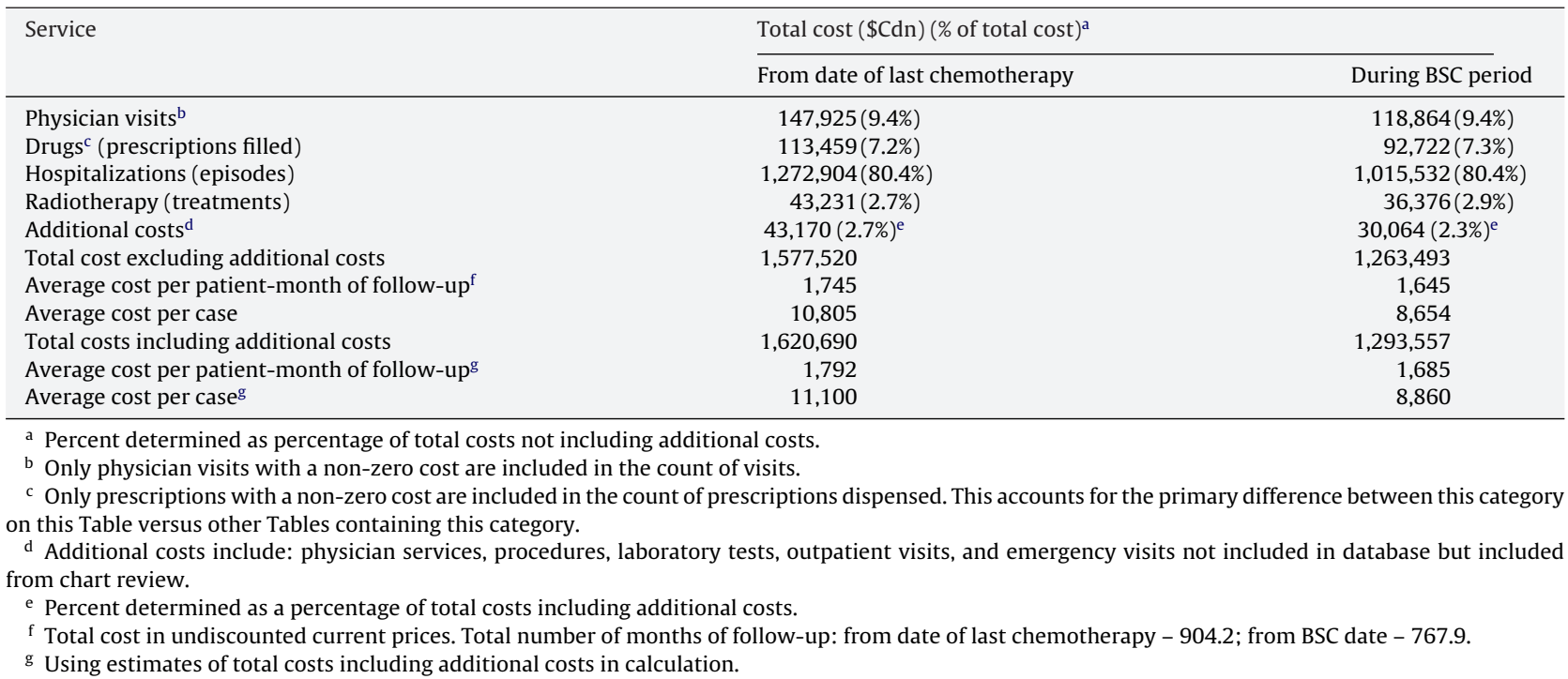




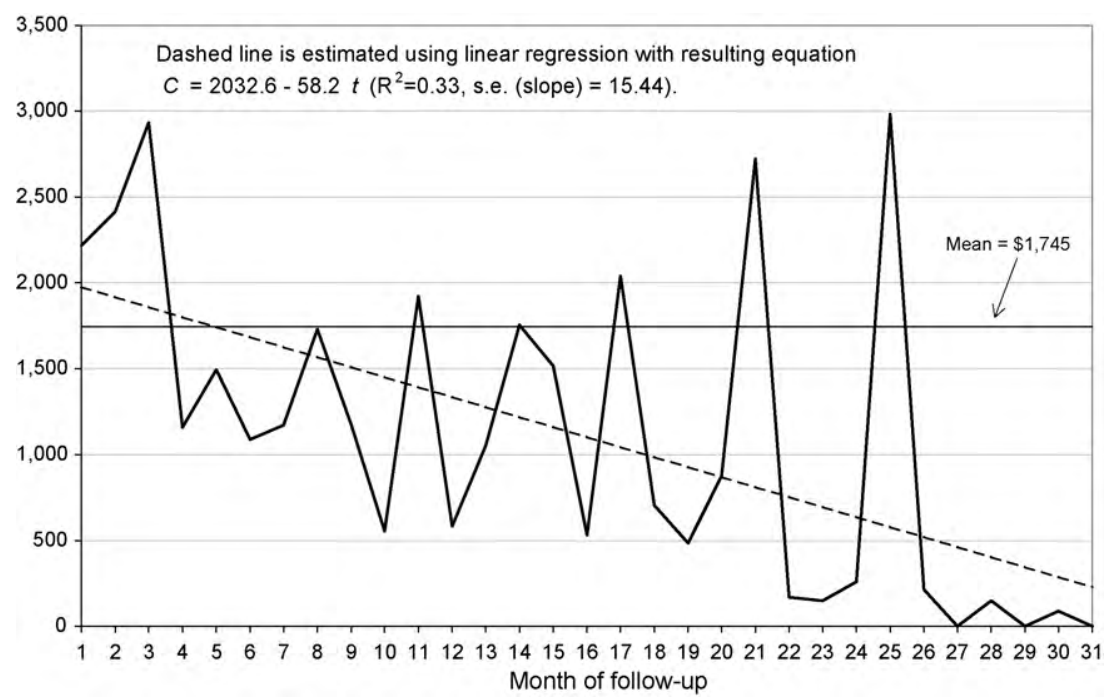

Fig. 1. Average cost per patient-month by month of follow-up from date of last chemotherapy, current prices (undiscounted).

two values $(\$ 314,027)$ is explained by the 28 additional days of follow-up when the last date of chemotherapy is used. When the services captured through the chart review (such as physician services, procedures, laboratory tests, drugs, outpatient visits, and emergency visits) are added, the total cost increased by $2.3-2.7 \%$ $(\$ 1,577,520-\$ 1,620,690$ and $\$ 1,263,493-\$ 1,293,557)$.

The average cost per patient was $\$ 10,805$, and average cost per patient-month was $\$ 1745$ from the date of last chemotherapy. The average cost per patient was $\$ 8654$ and the average cost per patient-month was $\$ 1654$ during the BSC period (Table 5). Fig. 1 shows the monthly average costs per patients for the follow-up period. There was a significant decline in the cost with longer time of follow-up.

These additional costs are also used in calculations of "low" and "high" estimates of cost per patient-month in terms of current and constant prices. Four estimates are calculated for each cost statistic and these are presented in Table 6. Table 6 represents cost estimates in terms of current costs as there was little difference in the conversion of these costs using undiscounted current prices, constant (1998) prices undiscounted or constant (1998) prices discounted. For example, the low estimate of cost per patient-month from the date of last chemotherapy estimated using current prices (undiscounted) was $\$ 1745$. Using constant (1998) prices undiscounted, the cost becomes $\$ 1700$. Lastly, using constant prices (1998) prices discounted, the cost is estimated to be $\$ 1698$. The difference between these estimates is marginal.

Lifetime treatment cost estimates were obtained by applying values determined using survival analysis methods to the average costs per patient-month (Table 6). Conversion of lifetime costs to discounted prices had a small effect. However, there was a

Table 6

Estimates of treatment costs.

\begin{tabular}{lll}
\hline & $\begin{array}{l}\text { From date of last } \\
\text { chemotherapy }\end{array}$ & $\begin{array}{c}\text { During BSC } \\
\text { period }\end{array}$ \\
\hline $\begin{array}{l}\text { Low estimates of cost per patient-month } \\
\text { Current prices, undiscounted }\end{array}$ & $\$ 1,745$ & $\$ 1645$ \\
$\begin{array}{l}\text { High estimates of cost per patient-month } \\
\text { Current prices, undiscounted }\end{array}$ & $\$ 1,792$ & $\$ 1684$ \\
$\begin{array}{l}\text { Low estimates of lifetime treatment costs } \\
\text { Current prices, undiscounted }\end{array}$ & $\$ 10,770$ & $\$ 8702$ \\
$\begin{array}{l}\text { High estimates of lifetime treatment costs } \\
\text { Current prices, undiscounted }\end{array}$ & $\$ 11,057$ & $\$ 8902$ \\
\hline
\end{tabular}

$19 \%$ decrease in the lifetime treatment cost using current prices when calculated from the date of BSC compared to the date of last chemotherapy.

\section{Discussion}

This study defines the outcomes, treatment, and associated costs of Stage IIIb and IV NSCLC patients who completed chemotherapy, survived at least 28 days after receiving their last chemotherapy treatment, and did not receive any chemotherapy while on BSC. In contrast to other studies, this population-based investigation itemizes the services that accrue and are utilized after chemotherapy is completed until death and their associated costs.

Of the 2463 NSCLC cases diagnosed in the study period, at least $50 \%$ will have had advanced disease. A proportion of these patients who had good performance status and were able to tolerate combination chemotherapy may have benefited from chemotherapy as the primary treatment modality. However, only 6\% (150) of these patients received chemotherapy, which appears to be lower than expected. Chemotherapy for treatment of NSCLC was introduced in 1995 after a meta-analysis demonstrated a 5\% survival benefit at one year [11]. Our study period started two years after this publication, and hence, the new practice may not have been fully implemented in the early part of the study. This may partly explain the small number of patients that received chemotherapy in our population.

The median survival time of our patients was 40.6 weeks from the date of diagnosis and 13.8 weeks from BSC date. The one- and two-year survivals were $31 \%$ and $12 \%$, respectively. Direct comparison with other published studies is hazardous as most of them have strict patient inclusion criteria (randomized controlled trials) or unique treatment regimens [12-14]. However, with this in mind, Schiller et al. showed that NSCLC patients on platinum-based chemotherapies had a median survival time of 36 weeks [14]. If we consider the date of randomization to be equivalent to the first date of chemotherapy, our median survival of 32 weeks was comparable to that of Schiller et al.

The majority of services that were utilized by the patients are physician visits (medical claims) and prescriptions. As all the medical claims from the time of last chemotherapy were included, some of them may not be related to lung cancer. Morphine and codeine were the most prescribed drugs. Pain and shortness of breath are symptoms experienced by the majority of late-stage lung cancer patients, which certainly contributed to the high use of narcotics. 
However, not all of the prescriptions were related to lung cancer. For example, $11.5 \%$ of the 3050 prescriptions were for cardiovascular drugs. This may reflect the fact that lung cancer patients are generally elderly and smokers with associated co-morbidities.

Most importantly, while there were only 1.9 hospitalizations per person, they accounted for $80 \%$ of the health care costs from the time of completion of chemotherapy until death. This suggests that therapeutic measures decreasing the number or length of hospitalization could decrease costs of treating NSCLC patients. Adding the costs of the services abstracted for the chart review increased the total costs by only $2.3-2.7 \%$. This indicates that comprehensive administrative databases are available and can be effectively used for this kind of investigation, at least in Manitoba. In this study, the services and the costs were calculated from the last day of chemotherapy and during the BSC period. Approximately $30 \%$ of the health services used were within 28 days of the last date of chemotherapy. This is likely contributed by the chemotherapy follow-up visits and services for the management of chemotherapy complications. This was translated to a $19 \%$ difference (less) in the lifetime cost if we calculate the cost only for BSC period.

Ramsey et al. reported that expenditures for all services were increased for individuals receiving second- and third-line chemotherapy [13]. We can consequently expect greater costs in the future for treating NCSLC patients as more expensive anticancer drugs are marketed. However, one could argue that if these agents improve the quality of life and reduce hospital admissions, the total cost of care may diminish.

It is difficult to compare the results of this study with other published studies as none included patients that survived 28 days after their chemotherapy and followed them up until death. Many studies describe the costs of treatment of NSCLC as part of clinical trials and mainly focus on the cost of active treatment or studies evaluate costs of two arms of a randomized clinical trial, for example, BSC versus specific treatment in clinical trial $[15,16]$. However, Evans et al. used population-based modeling to determine that the total care cost for Stage IV cases was $\$ 16,501$ (1988 Canadian dollars) [17]. Approximately $60 \%$ of the cost they reported was related to terminal care of the patients. The conversion of these figures, using the Canadian Consumer Price Index (CPI) results in a cost of $\$ 21,196$ for Stage IV patients. Altogether, these values are higher than the lifetime treatment costs of \$8702-\$11,057 estimated in the present study. This may be explained by the differences in the methodology used. Our results are likely to be more accurate since the cost analysis is based on itemization of all the services patient received. Our study was carried out prior to the introduction of targeted therapy as second or third line option for advanced NSCLC and thus the study is not a reflection of the current practice which may be a limitation of the study. However, as we are interested in a similar cost analysis on patients treated with erlotinib, a targeted therapy, the current study will be used as a historical control to analyze the cost effectiveness of these agents in a population setting.

Comparison of our results with European and other international studies on NSCLC (non-Canadian studies) is again not quite valid due to differences in healthcare funding, different lung cancer treatment guidelines, and overall study design [18]. However, some recent studies and their findings deserve mention. In a study by Pompen et al. [19] direct costs associated with the management of patients with advanced NSCLC in The Netherlands were estimated. Similar to our study, this study conducted a retrospective chart review collecting data on treatment and costs from the date of diagnosis to the time of death. The average total cost was $€ 31,187$ and hospitalization was the major contributor to costs. Forty-four to $55 \%$ of the total cost was due to hospitalizations compared to $80 \%$ in our study. The second major constituent of cost in this study was chemotherapy. In another study from France [20], direct costs for treatment of patients with lung cancer were analyzed from first recurrence to death. Data was limited to 71 NSCLC patients where the average cost per patient was $€ 13,969$ with the highest contributor being terminal care costs $(51 \%)$ and second highest being chemotherapy (30\%). This study from France did not further itemize terminal care costs, however, hospitalizations can be considered to be a major contributor as all 71 NSCLC patients were in the hospital with an average of two admissions per patient. Lastly, in a recent study from Switzerland [21], patterns and costs of lung cancer treatment and management were examined in a sample of 85 NSCLC patients. The total cost for each NSCLC patient was reported as $€ 19,692$ with hospitalizations being the major contributor $(71 \%$ of cost). Chemotherapy was the second largest contributor of cost. Therefore, while costs as determined in our study are not directly comparable to those in the above European studies, a very common finding in these types of cost analysis in centers in Canada and Europe is that hospital admissions are a major factor in the total cost.

While the costs associated with the treatment of individual BSC lung cancer patients may not be substantial, the disease has a high incidence and therefore, its impact on the health care system is large. These figures will also change as more costly treatments become available. This study also clearly identifies a role for the development of new therapies that increase survival while decreasing episodes of hospitalizations, which is the main constituent of the total cost.

\section{Conflict of interest}

This study was supported by funding from Astra-Zeneca Canada Inc.

\section{References}

[1] Parkin DM. Global cancer statistics in the year 2000. Lancet Oncol $2001 ; 2: 533-43$

[2] Sher T, Dy WK, Adjei AA. Small cell lung cancer. Mayo Clin Proc 2008;83:355-67.

[3] Travis WD, Travis LB, Devera SS. Lung cancer. Cancer 1995;75:191-202.

[4] Franeschi S, Bidoli E. The epidemiology of lung cancer. Ann Oncol 1999;10:S3-6

[5] Stinchcombe TE, Lee CB, Socinski MA. Current approaches to advanced stage non-small cell lung cancer: first line therapy in patients with good functional status. Clin Lung Cancer 2006;4(7 Suppl.):S111-7.

[6] Soquet PJ, Chauvin F, Boissel JP, Cellerino R, Cormier Y, Ganz PA, et al. Polychemotherapy in advanced non-small cell lung cancer, a meta-analysis. Lancet 1993;342:19-21.

[7] Stinchcombe TE, Socinski MA. Considerations for second-line therapy of nonsmall cell lung cancer. The Oncologist 2008;13(Suppl. 1):28-36.

[8] Felip E, Rosell R. Pemetrexed as second-line therapy for advanced non-small cell lung cancer (NSCLC). Ther Clin Risk Manage 2008;4:579-85.

[9] Shepherd FA, Pereira JR, Ciuleanu T, Tan EH, Hirsh V, Thongprasert S, et al. Erlotinib in previously treated non-small cell lung cancer. N Engl J Med 2005;353:123-32.

[10] Carlson JJ, Veenstra DL, Ramsey SD. Pharmacoeconomic evaluations in the treatment of non-small cell lung cancer. Drugs 2008;68:1105-13.

[11] Non-Small Cell Lung Cancer Collaborative Group. Chemotherapy in non-small cell lung cancer. A meta-analysis using updated data on individual patients from 52 randomized clinical trials. BMJ 1995;311:899-909.

[12] Shepherd FA, Dancey J, Ramlau R, Mattson K, Gralla R, O’Rourke M, et al Prospective randomized trial of docetaxel versus best supportive care in patients with non-small cell lung cancer previously treated with platinumbased chemotherapy. J Clin Oncol 2000;18:2095-103.

[13] Ramsey SD, Martins RG, Blough DK, Tock LS, Lubeck D, Reyes CM. Second-line and third-line chemotherapy for lung cancer: Use and Cost. Am J Manage Care 2008;14:297-306.

[14] Schiller JH, Harrington D, Belani C, Langer C, Sandler A, KrookJ, et al. Comparison of four chemotherapy regimens for advanced non-small-cell lung cancer. N Engl J Med 2002;346:92-8.

[15] Ng R, Hasan B, Mittmann N, Florescu M, Shepherd FA, Ding K, et al. Economic analysis of NCIC CTG JBR.10: A randomized trial of adjuvant vinorelbine plus cisplatin compared with observation in early stage non-small-cell lung cancer-A report of the working group on economic analysis, and the lung disease site group, National Cancer Institute of Canada Clinical Trials Group. J Clin Oncol 2007;25:2256-61.

[16] Leighl N, Shepherd FA, Kwong R, Burkes RL, Feld R, Goodwin PJ. Economic analysis of the TAX 317 trial: Docetaxel versus best supportive care as second-line therapy of advanced non-small-cell lung cancer. J Clin Oncol 2002;20:1344-52. 
[17] Evans WK, Will BP, Berthelot JM, Wolfson MC. The cost of managing lung cancer in Canada. Oncology 1995;11(9 Suppl.):147-53.

[18] Molinier L, Combescure C, Chouaïd C, Daurès JP, Housset B, Fabre D, et al. Cost of lung cancer-a methodological review. Pharmacoeconomics 2006;24:651-9.

[19] Pompen M, Gok M, Novak A, van Wuijtswinkel R, Biesma B, Schramel F, et al. Direct costs associated with the disease management of patients with unresectable advanced non-small cell lung cancer in The Netherlands. Lung Cancer 2009;64:110-6.
[20] Braud A-C, Levy-Piedbois C, Piedbois P, Piedbois Y, Livartovski A, Le Vu B, et al. Direct treatment costs for patients with lung cancer from first recurrence to death in France. Pharmacoeconomics 2003;21:671-9.

[21] Dede KJ, Szucs TD, Bodes S, Joerger M, Lowy A, Russi EW, et al. Management and costs of treating lung cancer patients in a University Hospital. Pharmacoeconomics 2004;22:435-44. 